\title{
Etiology of Ageratum Yellow Vein Diseases in South China
}

Xiaoyang Jiao, Huanran Gong, Xuejian Liu, Yan Xie, and Xueping Zhou, State Key Laboratory of Rice Biology, Institute of Biotechnology, Zhejiang University, Hangzhou, Zhejiang 310058, China

\begin{abstract}
Jiao, X., Gong, H., Liu, X., Xie, Y., and Zhou, X. 2013. Etiology of Ageratum yellow vein diseases in South China. Plant Dis. 97:1497-1503.

Ageratum conyzoides is a common weed in agricultural regions in Asia. A. conyzoides plants exhibiting yellow vein symptoms were collected from Yunnan and Guangxi provinces of China. Polymerase chain reaction detection and sequence analysis showed that samples collected from Yunnan were mainly infected by Tobacco curly shoot virus (TbCSV) associated with Ageratum yellow vein China betasatellite (AYVCNB), while samples from Guangxi were mostly infected by Papaya leaf curl China virus (PaLCuCNV) and AYVCNB, or by Ager-

exhibiting dual infections by PaLCuCNV, AYVCNV, and AYVCNB. Agrobacterium-mediated inoculation of infectious clones showed that both TbCSV and AYVCNB or PaLCuCNV and AYVCNB produced typical yellow vein symptoms in A. conyzoides. Consequently, Ageratum yellow vein diseases in Yunnan and Guangxi provinces were caused by TbCSV/AYVCNB, PaLCuCNV/AYVCNB, or AYVCNV/ AYVCNB. The implications of these results in relation to the prevalence of begomoviruses in cultivated plants are discussed.
\end{abstract} atum yellow vein China virus (AYVCNV) and AYVCNB, with a few
Geminiviruses are plant-pathogenic viruses with single-stranded DNA (ssDNA) genomes encapsidated in twinned quasi-isometric particles. They cause severe diseases in major crop plants worldwide (11). Based on insect vectors, host ranges, and genome structures, the family Geminiviridae has been divided into four genera, Mastrevirus, Curtovirus, Topocuvirus, and Begomovirus (4). The viruses of the genus Begomovirus, which account for the largest number of species in the family, infect dicotyledonous plants. Most begomoviruses have bipartite genomes, designated as DNA-A and DNA-B components, while many species have only a single genomic component which is homologous to DNA-A of bipartite begomoviruses $(3,11)$.

Within the last few years, many monopartite begomoviruses have been found to be associated with betasatellites (formerly known as DNA $\beta$ ). The representative betasatellites are Ageratum yellow vein betasatellite (AYVB) and Cotton leaf curl Multan betasatellite (CLCUMB), which are essential for typical disease symptoms of Ageratum yellow vein disease (AYVD) and Cotton leaf curl disease (CLCuD), respectively $(1,17)$. Betasatellites are approximately half the size of the viral genome and have little sequence identity with viral genomic DNA; they depend on the helper viruses for replication, encapsidation, movement in plants, and transmission between plants. The $\beta \mathrm{C} 1$ protein encoded by the complementary-sense strand of betasatellites has been demonstrated to be a symptom determinant (6).

The study presented here shows that the Ageratum yellow vein China betasatellite (AYVCNB) is necessary for inducing typical yellow vein disease symptoms when co-infected with Tobacco curly shoot virus (TbCSV), Papaya leaf curl China virus (PaLCuCNV), or Ageratum yellow vein China virus (AYVCNV) in Ageratum conyzoides.

Corresponding author: Yan Xie, E-mail: xiey@zju.edu.cn

* The $\boldsymbol{e}$-Xtra logo stands for "electronic extra" and indicates that Figures 1 and 2 appear in color online.

Accepted for publication 6 June 2013.

http://dx.doi.org/10.1094/PDIS-01-13-0074-RE

(C) 2013 The American Phytopathological Society

\section{Materials and Methods}

1. Viral isolates. A. conyzoides plants exhibiting yellow vein disease symptoms were collected in fields from the cities of Baoshan, Dehong, Tengchong, and Xishuangbanna in Yunnan Province, and Nanning in Guangxi Province, China from 2002 to 2012 (Table 1).

2. DNA extraction and sequence analysis. Total nucleic acids were extracted from field-collected leaf samples as described (20). The degenerate primer pair PA and PB was used to amplify a fragment approximately $500 \mathrm{bp}$ in size, which partially covered the intergenic region (IR) and the $V 1$ (CP) gene of the genome (24). The specific primer pairs AYVCNVF/AYVCNVR for AYVCNV, Y1F1/Y6R2 for TbCSV, HeNfullF/HeNfullR for PaLCuCNV, and TbLCYNV-F/TbLCYNV-R for Tobacco leaf curl Yunnan virus (TbLCYNV) were designed to amplify the corresponding begomoviruses, respectively. The primer pairs $\mathrm{Hn} 2 \mathrm{~F} / \mathrm{Hn} 2 \mathrm{R}, \mathrm{Y} 35 \mathrm{~F} / \mathrm{Y} 35 \mathrm{R}$, and HeNfullF/HeNfullR were used for amplification of the full length of AYVCNV, TbCSV, and PaLCuCNV, respectively. Betasatellites were amplified with the universal primer pair beta01/ beta02 (23), while a specific primer pair AYVCNBF/ AYVCNBR was used for AYVCNB. The polymerase chain reaction (PCR) products were cloned and sequenced by an automated $\mathrm{ABI}$ sequencer model 3730 (Perkin Elmer, Wellesley, MA, USA). The sequences of primers used for PCR detection are shown in Table 2. Sequences were assembled with the aid of Lasergene (DNASTAR Inc., Madison, WI, USA). Multiple sequence alignments were performed using MegAlign in Lasergene.

3. Infectious clones and agroinoculation of plants. The infectious clones of TbCSV (pBinplus-Y35-1.9A), PaLCuCNV (pBinplusHeNZM1-1.4A), AYVCNV (pBinplus-Hn2-1.4A), TbLCYNV (pBinplus-Y143-1.5A), AYVCNB (pBinplus-Hn19-2 $\beta$ ), and Tobacco curly shoot betasatellite (TbCSB) (pBinplus-Y35 $\beta-2.0)$ used in this study were reported previously $(13,19,21,22)$. The overnight Agrobacterium tumefaciens cultures containing the corresponding constructs were pelleted and resuspended in infiltration buffer containing $10 \mathrm{mM}$ MES ( $\mathrm{pH} 5.5$ ), $10 \mathrm{mM} \mathrm{MgCl} 2$, and $100 \mu \mathrm{M}$ acetosyringone and adjusted to an $\mathrm{OD}_{600}$ volume of 1.0. Nicotiana benthamiana and A. conyzoides seedlings in the four- to six-leaf stages were inoculated with viruses by injecting on the stems using a 1-ml syringe. The inoculated plants were placed in an insect-free growth chamber at $25^{\circ} \mathrm{C}$ with supplementary lighting to provide a 16-h photoperiod.

4. Southern blot hybridization. Total nucleic acids were extracted from young leaves of $N$. benthamiana and A. conyzoides 
Table 1. Information on Ageratum conyzoides collection locations, years, and detection of begomoviruses and betasatellites

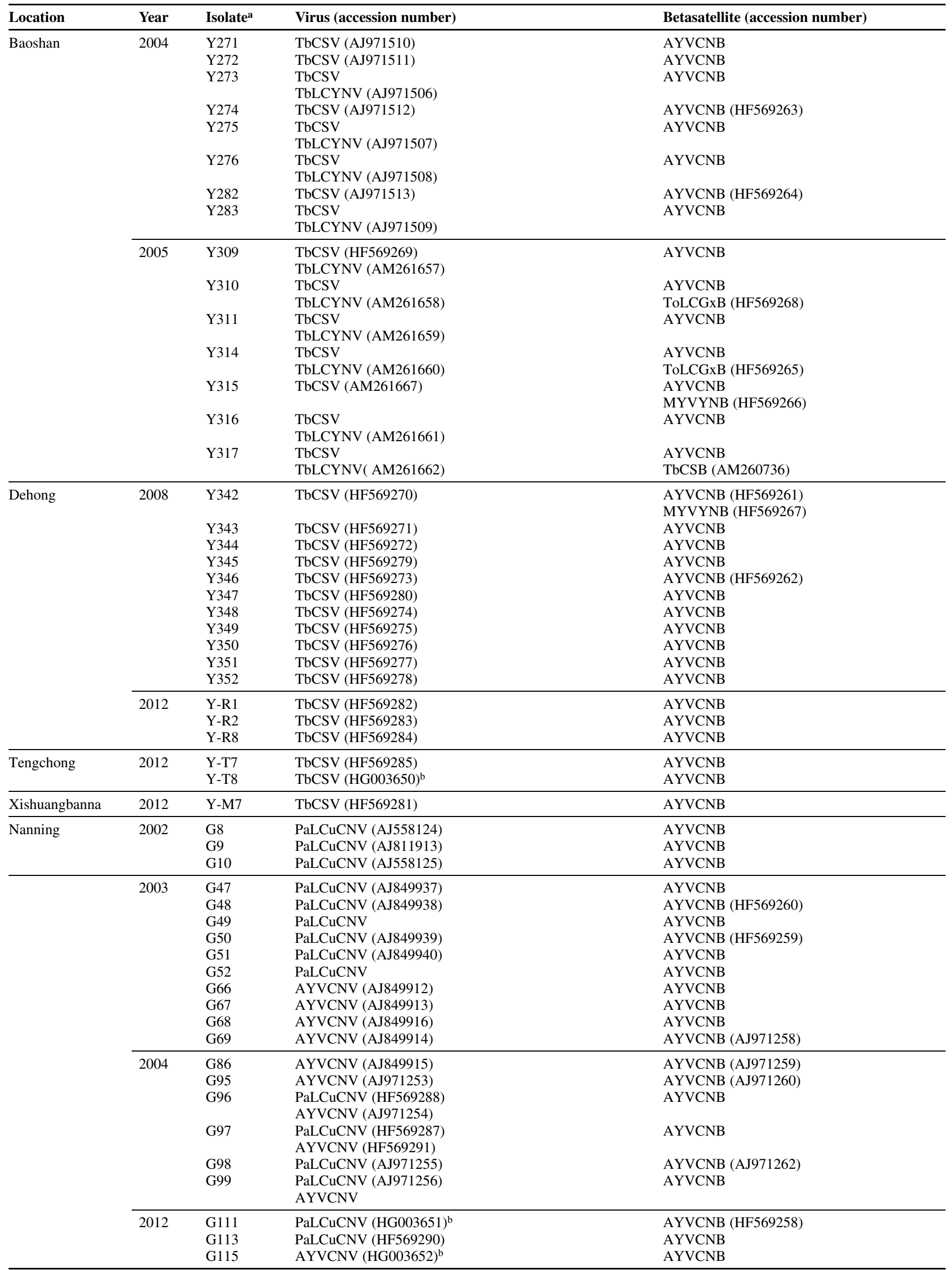

\footnotetext{
${ }^{a}$ Isolates begining with $\mathrm{Y}$ and $\mathrm{G}$ were collected from Yunnan Province and Guangxi Province, respectively.

${ }^{b}$ Accession numbers for the full-length genome.
} 
plants using the CTAB method as described (20). Nucleic acids were fractionated on $1 \%$ agarose gel in TBE buffer $(90 \mathrm{mM}$ Trisborate, 2 mM EDTA, $\mathrm{pH} 8.3$ ) and transferred to Hybond- ${ }^{+}$membranes (Amersham Biosciences, Buckinghamshire, England) and hybridized with Digoxin-labeled probes specific for AYVCNV, TbCSV, PaLCuCNV, or AYVCNB using a DIG High Prime DNA Labeling and Detection Starter Kit I (Roche Diagnostics, Indianapolis IN, USA).

\section{Results}

Detection of begomoviruses from samples collected in South China. Using the degenerate primer pair $\mathrm{PA} / \mathrm{PB}$, a fragment of about $500 \mathrm{bp}$ was amplified from all the collected A. conyzoides plant samples. The amplified fragments were cloned and then sequenced. Sequence similarity searches were performed using the Basic Local Alignment Search Tool (BLAST) algorithm available in GenBank, and the results showed that most of the A. conyzoides samples isolated from Yunnan (Y271-272, Y274, Y282, Y315, Y342-Y352, Y-R1-R2, Y-T7-T8, Y-R8, Y-M7) had the highest sequence similarities (more than 93.0\%) with TbCSV, while some samples (Y273, Y275-276, Y283, Y309-311, Y314, Y316-317) had more than $96.2 \%$ sequence identities with TbLCYNV (Table 1). The TbCSV-specific primer pair Y1F1/Y6R2 and TbLCYNVspecific primer pair TbLCYNV-F/TbLCYNV-R were then used to amplify the putative product from the DNA extracted from all the 32 Yunnan samples. The specific 1.0-kb fragment of TbCSV was generated from all A. conyzoides samples collected in Yunnan, including samples shown to be infected with TbLCYNV, while the specific 1.0-kb fragment of TbLCYNV was only detected in Y273, Y275-276, Y283, Y309-311, Y314, Y316, and Y317 samples, suggesting that all $A$. conyzoides samples collected in Yunnan were infected by $\mathrm{TbCSV}$, and that some samples were dually infected by both TbCSV and TbLCYNV (Table 1). Y-T8 was randomly selected for the purpose of amplifying its entire genome using $\mathrm{Y} 35 \mathrm{~F} / \mathrm{Y} 35 \mathrm{R}$ primers, and fully sequenced, which revealed that it shared $96.1 \%$ sequence identity with TbCSV.

Amplified fragments from A. conyzoides plant samples in Guangxi Province were also sequenced. Sequence analyses showed that $A$. conyzoides samples collected in Guangxi could be clearly classified into two groups. G8-10, G47-52, G97-99, G111, and G113 were most closely related to PaLCuCNV with sequence identity exceeding 94.0\%, while G66-69, G86, G95-96, and G115 each exhibited more than $90.8 \%$ identity with AYVCNV. These findings were successfully validated by using the specific primers HeNfullF/HeNfullR for PaLCuCNV and AYVCNVF/AYVCNVR for AYVCNV to detect the respective viruses in all 22 samples isolated from Guangxi. A specific band of PaLCuCNV approximately $2.7 \mathrm{~kb}$ in size was amplified from the samples G8-10, G4752, G96-99, G111, and G113. G111 was then chosen for the full genome cloning and sequencing, which revealed that it shared $93.4 \%$ sequence identity with PaLCuCNV. A 500-bp fragment of AYVCNV was obtained from the samples G66-69, G86, G95-97, G99, and G115. G115 was amplified with the primers $\mathrm{Hn} 2 \mathrm{~F} / \mathrm{Hn} 2 \mathrm{R}$ by PCR, and sequence analysis showed that its full-length DNA sequence shared $90.1 \%$ identity with that of AYVCNV. Additionally, both AYVCNV and PaLCuCNV were detected in G96-97 and G99.

Detection of betasatellites from samples collected in South China. Betasatellites were detected in all A. conyzoides plants by PCR using the abutting primer pair beta01/beta02. Only five of the 32 samples from Yunnan yielded the specific $1.3-\mathrm{kb}$ fragment. The PCR fragments were cloned and then sequenced, and sequence analyses showed that Tomato leaf curl Guangxi betasatellite (ToLCGxB) existed in samples Y310 and Y314, Malvastrum yellow vein Yunnan betasatellite (MYVYNB) was detected in samples Y315 and Y342, and TbCSB was found in sample Y317 (Table 1). A previous study demonstrated that betasatellites are essential for the induction of typical yellow vein symptoms for AYVD (17) and that severe yellow vein symptoms were only produced after coinoculation with both AYVCNV and AYVCNB (21). Accordingly, the specific primer pair AYVCNBF/AYVCNBR for detection of AYVCNB was used to screen all the Yunnan samples. As a result, a specific band of approximately $1.3 \mathrm{~kb}$ was consistently amplified from all $A$. conyzoides plants. The amplified PCR fragments from isolates of Y274, Y282, Y342, and Y346 were randomly selected for cloning and sequencing, and sequence analyses revealed that the nucleotide sequences of betasatellites from these isolates were most closely related to AYVCNB ( $>86.2 \%$ nucleotide sequence identity). These data suggested that the predominant betasatellite associated with $A$. conyzoides plants in Yunnan was AYVCNB.

In an attempt to detect betasatellite components from $A$. conyzoides plant samples in Guangxi, the specific primers AYVCNBF/AYVCNBR for AYVCNB and the abutting primers beta01/beta02 were used to amplify the potential betasatellites. PCR revealed that all the samples from Guangxi were associated with AYVCNB (Table 1). The amplified PCR fragments from isolates G48, G50, G69, G86, G95, G98, and G111 were randomly selected for cloning and sequencing; sequence analyses revealed that the full sequences of betasatellites obtained from these isolates shared more than $94 \%$ nucleotide sequence identity with that of AYVCNB. The above results show that plants from Guangxi were mostly infected by PaLCuCNV/AYVCNB, and seven plants were infected by the AYVCNV/AYVCNB complex, while three plants

Table 2. Primers used in polymerase chain reaction (PCR) analysis of samples for virus detection

\begin{tabular}{|c|c|c|}
\hline Primer & Sequence $\left(5^{\prime} \rightarrow 3^{\prime}\right)^{\mathrm{a}}$ & Position in genome \\
\hline \multicolumn{3}{|c|}{ For virus detection } \\
\hline PA & 5'-TAATATTACCKGWKGVCCSC-3' & $2762-13$ \\
\hline PB & 5'-TGGACYTTRCAWGGBCCTTCACA-3' & $544-522$ \\
\hline AYVCNVF & 5'-AAGTGGTCCCCACCACTAAC-3' & $28-47$ \\
\hline AYVCNVR & 5'-ACATGGGATATGTCGTGCCTCTG-3' & $555-577$ \\
\hline Y1F1 & 5'-CGTAGGCCTGTGGATAAACCTCAAGAT-3' & $720-746$ \\
\hline Y6R2 & 5'-GGAAGCCAGTTCAAATTAAAGG-3' & $1736-1715$ \\
\hline HeNfullF & 5'-GGGATCCTTTACTAAACGAGTTTCC-3' & $141-165$ \\
\hline HeNfullR & 5'-AGGATCCCACATGTTTGACGTG-3' & $148-127$ \\
\hline TbLCYNV-F & 5'-GATTGGTACCTGAGTACAATG-3' & $1566-1586$ \\
\hline TbLCYNV-R & 5'-TTATCCACAGTGCTCTCTCAC-3' & $2554-2534$ \\
\hline $\mathrm{Hn} 2 \mathrm{~F}$ & 5'-GTGGGATCCTCTTTTGAACGAG-3' & $139-160$ \\
\hline $\mathrm{Hn} 2 \mathrm{R}$ & 5'-AGAGGATCCCACATGTTTGGATTTAG-3' & $150-131$ \\
\hline $\mathrm{Y} 35 \mathrm{~F}$ & $5^{\prime}$-CTGGATCCATTAGTAAACGAGTTTC-3' & $139-161$ \\
\hline Y35R & 5'-TAGGATCCCACATAGTGCGGAGTGC-3' & $144-122$ \\
\hline \multicolumn{3}{|c|}{ For betasatellite detetion } \\
\hline beta 01 & 5'-GTAGGTACCACTACGCTACGCAGCAGCC-3' & $1274-1298$ \\
\hline beta 02 & 5'-AGTGGTACCTACCCTCCCAGGGGTACAC-3' & $1279-1255$ \\
\hline AYVCNBF & $5^{\prime}$ - CCGATAGGTAAATGAGACACCGATAC- $3^{\prime}$ & $1037-1062$ \\
\hline AYVCNBR & 5'- GGTGTCCAATTATTTACCTTGTGAGG-3' & $1013-1038$ \\
\hline
\end{tabular}

${ }^{\mathrm{a}} \mathrm{B}=\mathrm{C}, \mathrm{T}$, or $\mathrm{G} ; \mathrm{K}=\mathrm{G}$ or $\mathrm{T} ; \mathrm{R}=\mathrm{A}$ or $\mathrm{G} ; \mathrm{S}=\mathrm{C}$ or $\mathrm{G} ; \mathrm{V}=\mathrm{A}, \mathrm{C}$, or $\mathrm{G} ; \mathrm{W}=\mathrm{A}$ or $\mathrm{T} ; \mathrm{Y}=\mathrm{C}$ or $\mathrm{T}$. 
were dually infected by both PaLCuCNV and the AYVCNV/ AYVCNB complex.

Infectivity and symptoms associated with either the begomovirus or the begomovirus/betasatellite complex. To ascertain the etiology of AYVD in Yunnan and Guangxi, the infectious clones AYVCNV (pBin-Hn2-1.4A), TbCSV (pBinplus-Y35-1.9A), PaLCuCNV (pBinplus-HeNZM1-1.4A), and TbLCYNV (pBinPLUSY143-1.5A) were agroinoculated into $N$. benthamiana and $A$. conyzoides plants either alone or in association with AYVCNB (pBinplus-Hn19-2 $\beta$ ) or TbCSB (pBinplus-Y35 $\beta-2.0$ ). Using Agrobacterium-mediated inoculation into $N$. benthamiana, AYVCNV alone was found to be incapable of inducing any symptoms, although it could be detected in inoculated plants; but when coinoculated with AYVCNB, the severe downward leaf curling and vein thickening symptoms were observed (Fig. 1A and B). When

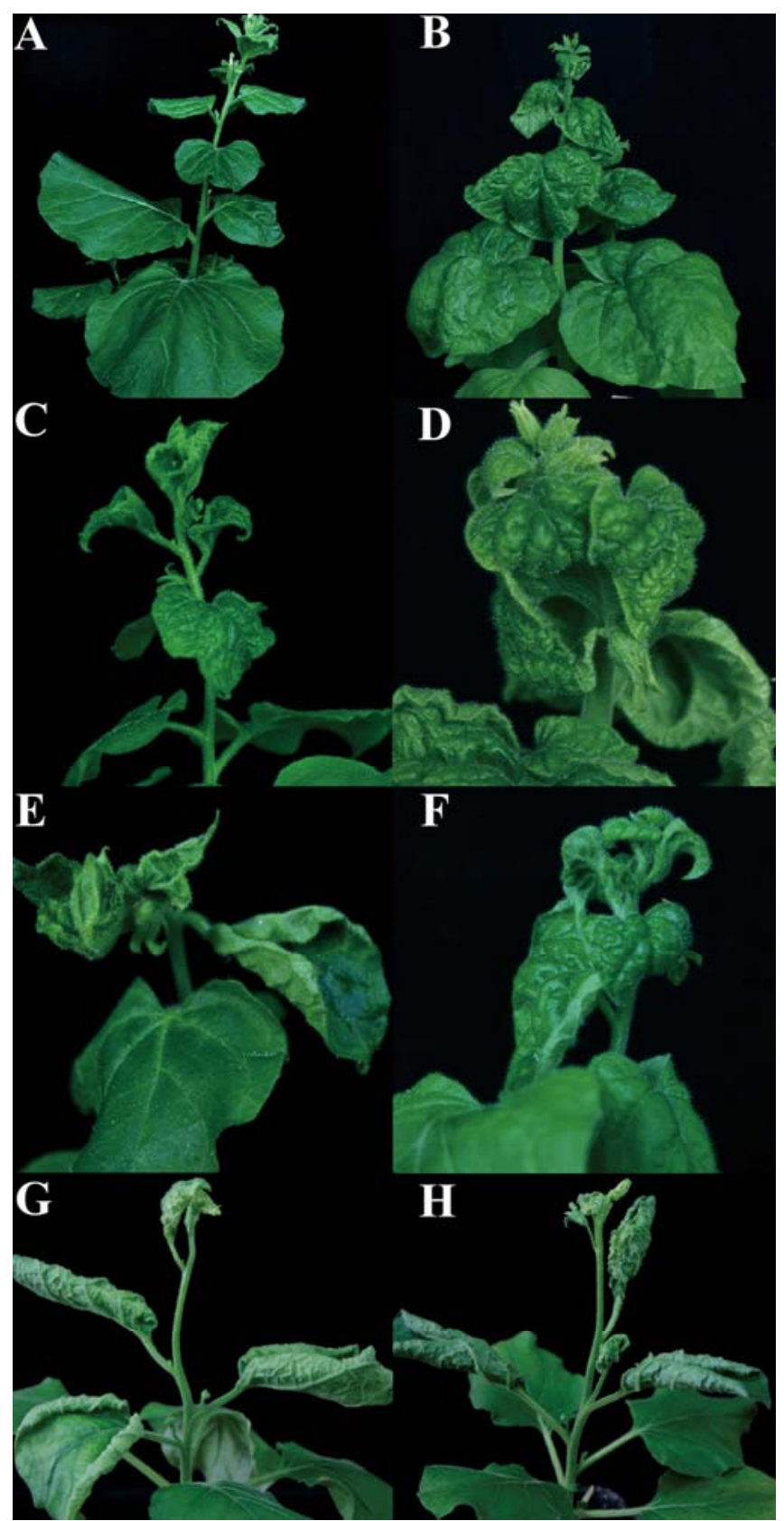

Fig. 1. Symptoms displayed by Nicotiana benthamiana plants induced with $\mathbf{A}$, Ageratum yellow vein China virus (AYVCNV) alone, B, AYVCNV and Ageratum yellow vein China betasatellite (AYVCNB), C, Tobacco curly shoot virus (TbCSV) alone, D, TbCSV and AYVCNB, E, Papaya leaf curl China virus (PaLCuCNV) alone, F, PaLCuCNV and AYVCNB, G, TbLCYNV alone, or H, TbLCYNV and AYVCNB at $30 \mathrm{dpi}$. agroinoculated with TbCSV alone, $N$. benthamiana plants produced upward leaf curling, vein thickening, and stunting symptoms; whereas severe downward leaf curling, vein thickening, and stunting symptoms were induced when co-inoculated with AYVCNB (Fig. $1 \mathrm{C}$ and $\mathrm{D})$. PaLCuCNV alone could induce severe upward leaf curling and vein thickening symptoms, while severe downward leaf curling and vein thickening symptoms developed after 7 days postinoculation (dpi) when co-inoculated with AYVCNB (Fig. 1E and F). All the infectious clones were highly pathogenic to $N$. benthamiana plants with $100 \%$ infection rates (Table 3 ).

In A. conyzoides, AYVCNV, TbCSV, or PaLCuCNV alone (Fig. 2A, D, and G), or TbCSV and TbCSB together produced asymptomatic infections. However, A. conyzoides plants co-inoculated with AYVCNB and one of these begomoviruses developed yellow vein symptoms that were indistinguishable from those of naturally infected plants (Fig. 2B, E, and H). More severe yellow vein disease symptoms developed within 70 dpi (Fig. 2C, F, and I). In contrast, TbLCYNV alone or in co-inoculation with AYVCNB could not induce symptoms in A. conyzoides, while it induced upward leaf curling and vein thickening symptoms in $N$. benthamiana (Table 3; Fig. 1G and $\mathrm{H}$ ).

Southern blot hybridization analysis was used to assess the accumulation of TbCSV, PaLCuCNV, AYVCNV, and AYVCNB in systemically infected leaves of inoculated plants. The accumulation of viral DNA of TbCSV and PaLCuCNV was readily detected in $N$. benthamiana plants inoculated with TbCSV or PaLCuCNV alone or during co-inoculation with AYVCNB. In A. conyzoides plants, the accumulation of viral DNA was detected at low levels after inoculations with TbCSV or PaLCuCNV alone, but was significantly higher in plants co-inoculated with AYVCNB. The accumulation of AYVCNB was easily detected in co-inoculated plants of both $N$. benthamiana and A. conyzoides (Fig. 3). AYVCNB enhanced the accumulation of viral DNA of TbCSV and $\mathrm{PaLCuCNV}$ in systemically infected leaves in A. conyzoides. The DNA accumulation levels of TbCSV and PaLCuCNV in systemically infected $N$. benthamiana plants were similar with or without AYVCNB. These results indicate that TbCSV or PaLCuCNV requires the presence of AYVCNB to induce typical yellow vein disease symptoms in A. conyzoides plants.

\section{Discussion}

Weeds are important intermediate hosts for geminiviruses and play a significant role in virus epidemiology in the field due to

Table 3. Infectivity and symptoms induced by different combinations of begomoviruses and betasatellites

\begin{tabular}{lccc}
\hline $\begin{array}{l}\text { Plant } \\
\text { species }\end{array}$ & Inoculum & $\begin{array}{c}\text { Plants infected/ } \\
\text { plants inoculated }\end{array}$ & Symptoms $^{\mathbf{a}}$ \\
\hline Nicotiana benthamiana & & \\
& AYVCNV & $4 / 4$ & NS \\
AYVCNV + AYVCNB & $6 / 6$ & DLC, VT \\
TbCSV & $6 / 6$ & ULC, VT, ST \\
TbCSV + TbCSB & $6 / 6$ & DLC, VT, ST \\
TbCSV + AYVCNB & $6 / 6$ & DLC, VT, ST \\
PaLCuCNV & $6 / 6$ & ULC, VT \\
PaLCuCNV + AYVCNB & $6 / 6$ & DLC, VT \\
TbLCYNV & $6 / 6$ & ULC, VT \\
TbLCYNV + AYVCNB & $6 / 6$ & ULC, VT \\
Ageratum conyzoides & & \\
AYVCNV & $6 / 6$ & NS \\
AYVCNV + AYVCNB & $26 / 26$ & YV \\
TbCSV & $24 / 26$ & NS \\
TbCSV + TbCSB & $24 / 25$ & NS \\
TbCSV + AYVCNB & $26 / 26$ & YV \\
PaLCuCNV & $25 / 26$ & NS \\
PaLCuCNV + AYVCNB & $26 / 26$ & YV \\
TbLCYNV & $0 / 25$ & NS \\
TbLCYNV + AYVCNB & $0 / 25$ & NS \\
\hline
\end{tabular}

${ }^{\text {a DLC, downward leaf curling; VT, vein thickening; ULC, upward leaf }}$ curling; ST, stunting; YV, yellow vein; NS, no symptoms. 
their survival ability and wide distribution. A. conyzoides is a widespread weed acting as a reservoir host for economically significant viral diseases $(8,9,12)$. In addition to AYVV associated with AYVB, which occurs widely in A. conyzoides throughout southeastern Asia (15), other begomoviruses are also progressively identified from A. conyzoides each year, such as the Ageratum yellow vein Hualian virus (AYVHuV) and the Ageratum yellow vein Sri Lanka virus (AYVSLV) (7). Recently, Tomato leaf curl Java virus (ToLCJV) and AYVV-[Java] recombinant virus named ToLCJV-Ageratum, and the associated betasatellite ToLCJV-Ageratum DNA $\beta 03$, were isolated from A. conyzoides in Indonesia (9). In Nepal, Pakistan, and India, A. conyzoides has exhibited infections by Ageratum enation virus (AEV) (10). In Cameroon, A. conyzoides was found to be infected with Ageratum leaf curl Cameroon virus (ALCCMV) accompanied by Ageratum leaf curl Cameroon betasatellite (ALCCMB) and Ageratum leaf curl Cameroon alphasatellite (ALCCMA) (12). AYVD is widespread in South China. AYVCNV accompanied by AYVCNB was observed in A. conyzoides plants in Haikou, Hainan Province (21). In addition to AYVCNV, Ageratum leaf curl virus (ALCV) was isolated from A. conyzoides in Guangxi Province (8). While begomoviruses alone have no ability to cause AYVD in A. conyzoides plants, only some betasatellites like AYVB, AYVCNB, or ToLCJV-Ageratum
DNA $\beta 03$ are required for the induction of yellow vein disease symptoms in $A$. conyzoides plants $(9,15,19)$.

In this paper, 54 field-grown $A$. conyzoides plants showing yellow vein symptoms were collected in Yunnan and Guangxi provinces from 2002 to 2012, and the sequence analyses showed that they were mostly infected by TbCSV/AYVCNB or PaLCuCNV/ AYVCNB complexes, rather than by an AYVCNV/AYVCNB complex. To fulfill Koch's postulates, the infectious clone assays were performed, which showed that the heterogeneous begomovirus complexes, TbCSV/AYVCNB or PaLCuCNV/AYVCNB, could induce the typically severe symptoms associated with yellow vein disease in A. conyzoides. PCR and Southern blot analyses confirmed that AYVCNB could interact successfully with TbCSV or PaLCuCNV in A. conyzoides. Therefore TbCSV/ AYVCNB and $\mathrm{PaLCuCNV/AYVCNB}$ were the predominant complexes found in A. conyzoides plants collected in Yunnan and Guangxi provinces, respectively. Given its unique microclimate, Yunnan possesses the greatest biodiversity among the provinces in China. This may explain the predominance of the two begomoviruses we observed in adjacent provinces. The abutting primers beta01/beta02 were used for amplification of betasatellites in A. conyzoides plants, and only a few samples yielded the specific $1.3-\mathrm{kb}$ fragment. When the specific primer pair AYVCNBF/AYVCNBR was used, AYVCNB
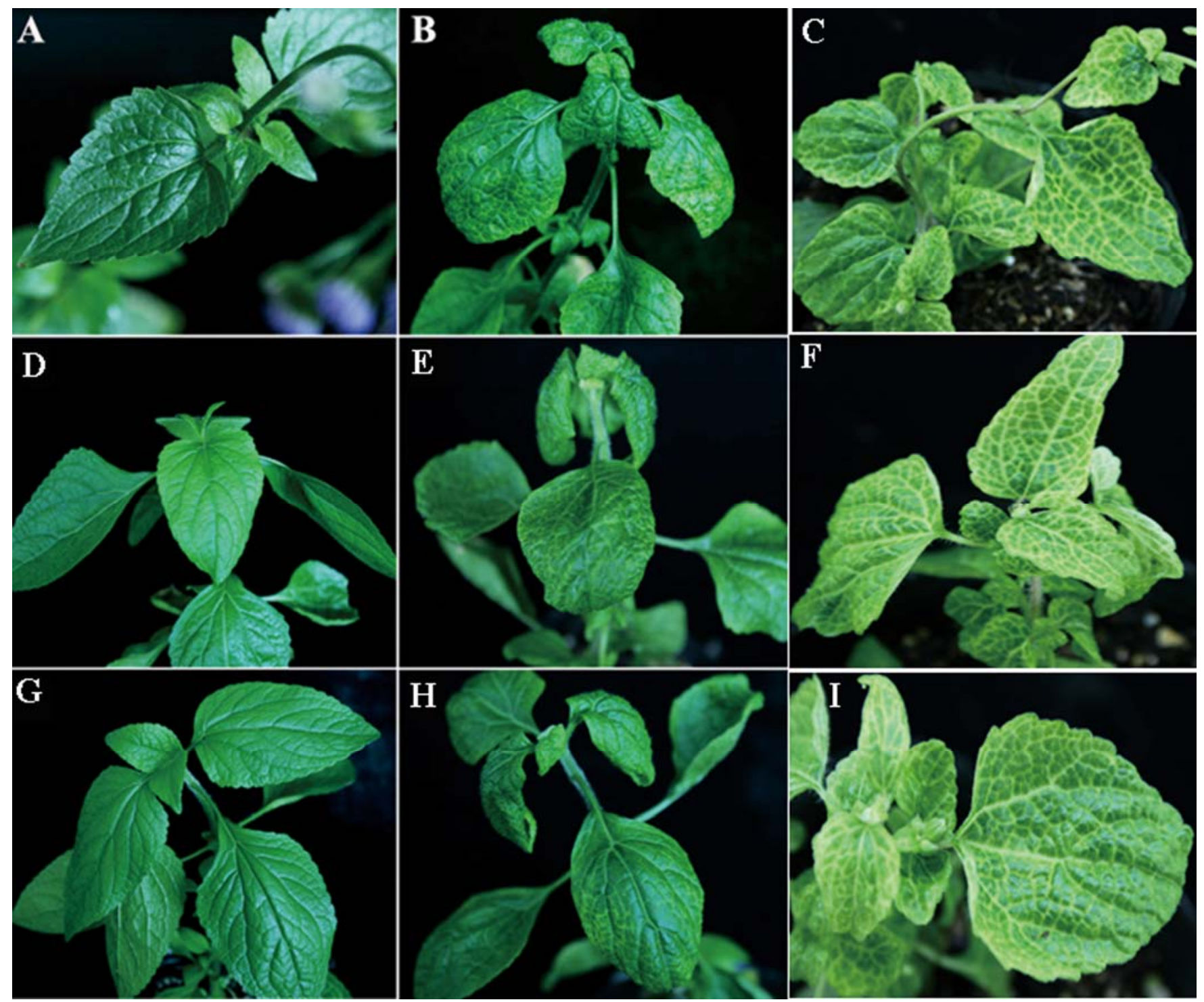

Fig. 2. Symptoms exhibited by Ageratum conyzoides plants induced by A, Ageratum yellow vein China virus (AYVCNV), D, Tobacco curly shoot virus (TbCSV), and G, Papaya leaf curl China virus (PaLCuCNV) alone at $50 \mathrm{dpi}$, or AYVCNV and Ageratum yellow vein China betasatellite (AYVCNB) at B, $50 \mathrm{dpi}$ and C, $70 \mathrm{dpi}$, or TbCSV and AYVCNB at E, 50 dpi and F, 70 dpi, or PaLCuCNV and AYVCNB at H, 50 dpi and I, 70 dpi. 


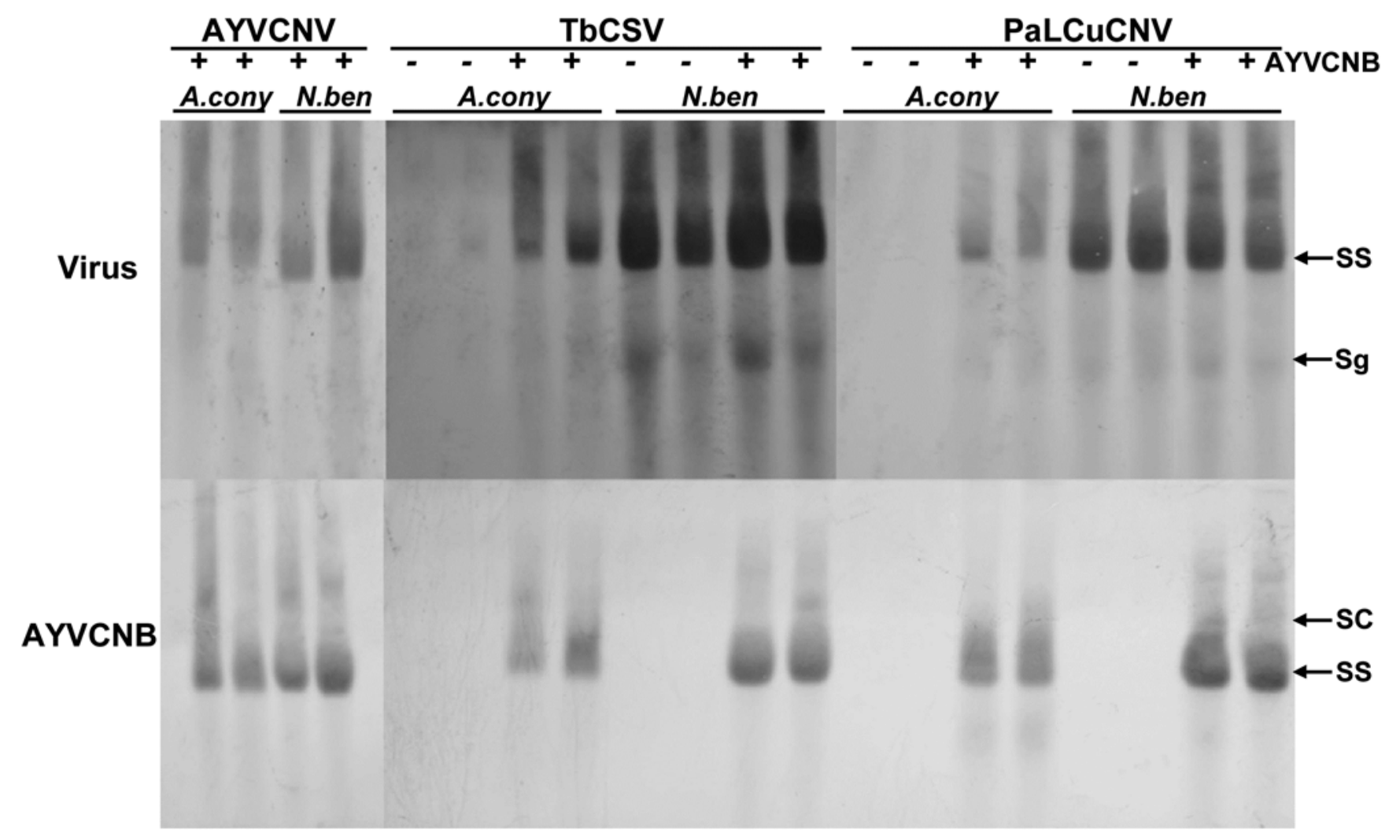

Fig. 3. Southern blot analysis of nucleic acid extracted from infected Nicotiana benthamiana and Ageratum conyzoides plants agroinoculated with Ageratum yellow vein China virus (AYVCNV), Tobacco curly shoot virus (TbCSV), or Papaya leaf curl China virus (PaLCuCNV) alone (-) or together with Ageratum yellow vein China betasatellite (AYVCNB) (+). Blots were probed with AYVCNV, TbCSV, and PaLCuCNV (upper) or AYVCNB (lower). Equal amount of total DNA extracts (approximately $30 \mu \mathrm{g}$ ) were loaded in each lane. Expected positions of single-stranded, supercoiled, and subgenomic DNA forms are labled as SS, SC, and Sg, respectively.

was detected in all samples. Sequence analysis of all the betasatellites indicated that the sequence of the beta02 primer is not present in the AYVCNB genome; this can explain why the abutting primers beta01 and beta02 are not suitable for PCR amplification of AYVCNB.

Surveys in China have found that there are three types of monopartite begomoviruses. Most monopartite begomoviruses, such as TbLCYNV and PaLCCNV, do not need betasatellites to infect host plants $(19,22)$. Many monopartite begomoviruses, such as Tomato yellow leaf curl China virus (TYLCCNV) and AYVCNV, are associated with betasatellites, and are completely dependent on the corresponding betasatellites for efficient infection of host plants and for symptom development $(5,21)$. Some monopartite begomoviruses like TbCSV are associated with betasatellites, while TbCSV is capable of infecting host plants and inducing disease symptoms in the presence or absence of a betasatellite (13). Betasatellite components have a relaxed specificity for begomoviruses during their replication (14). AYVV can support the replication and systemic spread of betasatellites associated with CLCuMV, Eupatorium yellow vein virus (EpYVV), and Honeysuckle yellow vein virus (HYVV) (16). AYVCNV can interact functionally with the heterologous betasatellite components associated with TYLCCNV, TbCSV, Malvastrum yellow vein virus (MYVV), and Sida yellow mosaic China virus (SiYMCNV) (21). Hence, begomoviruses trans-replicating heterologous betasatellites is a common phenomenon in nature. AYVCNV and other similar begomoviruses have the ability to trans-replicate several heterologous betasatellites but were unable to induce AYVD with these other betasatellites in A. conyzoides plants in our study. This is because of unique defensive mechanisms present in A. conyzoides.

$A$. conyzoides can be infected by begomoviruses and serves as a reservoir of viruses that are also pathogenic to cultivated plant species. The AYVV/AYVB complex was reported to be an emerging disease in cultivated tomato plants, causing tomato yellow leaf curl disease (TYLCD) in Japan (2). AYVB has the ability to associate with Tomato yellow leaf curl virus (TYLCV) to produce severe TYLCD, suggesting that reassortant viruses like TYLCV/ AYVB can potentially cause severe diseases in vegetables and ornamental crops (18). Our data showed that AYVCNB is necessary to induce severe yellow vein disease symptoms in $A$. conyzoides and could be trans-replicated by heterogeneous TbCSV or PaLCuCNV. TbCSV, a monopartite begomovirus associated with TbCSB, which is not necessary for causing infections in host plants, is widely distributed on tobacco and tomato plants in Yunnan Province, South China (13). On the other hand, PaLCuCNV, a monopartite begomovirus lacking betasatellite DNA, occurs widely on tobacco and tomato plants in Guangxi and Henan provinces in South China (22). TbCSV and PaLCuCNV are capable of acquiring the betasatellite AYVCNB from other begomovirus to form new reassortments, and thus expand their host range and cause prevalent yellow vein disease in A. conyzoides plants. These new reassortments in $A$. conyzoides plants might represent original inoculum with the potential to infect cultivated tobacco and tomato crops, thereby aggravating diseases caused by TbCSV and PaLCCNV in these crops. Therefore, to control these diseases, it is more important to eliminate any wild $A$. conyzoides plants growing near cultivated plants.

\section{Acknowledgments}

This work was supported by National Natural Science Foundation of China (Grants No. 31171814 and No. U1136606).

\section{Literature Cited}

1. Amin, I., Hussain, K., Akbergenov, R., Yadav, J. S., Qazi, J., Mansoor, S., Hohn, T., Fauquet, C. M., and Briddon, R. W. 2011. Suppressors of RNA silencing encoded by the components of the Cotton leaf curl Begomovirusbetasatellite complex. Mol. Plant-Microbe Interact. 24:973-983.

2. Andou, T., Yamaguchi, A., Kawano, S., Kawabe, K., and Ueda, S. 2012. Ageratum yellow vein virus isolated from tomato plants with leaf curl on Ishigaki Island, Okinawa, Japan. J. Gen. Plant Pathol. 76:287-291.

3. Briddon, R. W., Patil, B. L., Bagewadi, B., Nawaz-ul-Rehman, M. S., and Fauquet, C. M. 2010. Distinct evolutionary histories of the DNA-A 
and DNA-B components of bipartite begomoviruses. BMC Evol. Biol. 10:97.

4. Brown, J. K., Fauquet, C. M., Briddon, R. W., Zerbini, M., Moriones, E., and Navas-Castillo, J. 2012. Geminiviridae. Pages 351-373 in: Virus Taxonomy - Ninth Report of the International Committee on Taxonomy of Viruses. A. M. Q. King, M. J. Adams, E. B. Carstens, and E. J. Lefkowitz, eds. Associated Press, Elsevier Inc., London.

5. Cui, X. F., Tao, X. R., Xie, Y., Fauquet, C. M., and Zhou, X. P. 2004. A DNA $\beta$ associated with Tomato yellow leaf curl China virus is required for symptom induction. J. Virol. 78:13966-13974.

6. Ding, C. J., Qing, L., Li, Z. H., Liu, Y., Qian, Y. J., and Zhou, X. P. 2009. Genetic determinants of symptoms on viral DNA satellites. Appl. Environ. Microbiol. 75:5380-5389.

7. Fauquet, C. M., Briddon, R. W., Brown, J. K., Moriones, E., Stanley, J., Zerbini, M., and Zhou, X. P. 2008. Geminivirus strain demarcation and nomenclature. Arch. Virol. 153:783-821.

8. Huang, J. F., and Zhou, X. P. 2006. Molecular characterization of two distinct begomoviruses from Ageratum conyzoides and Malvastrum coromandelianum in China. J. Phytopathol. 154:648-653.

9. Kon, T., Kuwabara, K., Hidayat, S. H., and Ikegami, M. 2007. A begomovirus associated with ageratum yellow vein disease in Indonesia: Evidence for natural recombination between Tomato leaf curl Java virus and Ageratum yellow vein virus-[Java]. Arch. Virol. 152:1147-1157.

10. Kumar, Y., Hallan, V., and Zaidi, A. A. 2011. First report of Ageratum enation virus infecting Crassocephalum crepidioides (Benth.) S. Moore and Ageratum conyzoides L. in India. J. Gen. Plant Pathol. 77:214-216.

11. Lazarowitz, S. G. 1992. Geminiviruses - genome structure and gene-function. Crit. Rev. Plant Sci. 11:327-349.

12. Leke, W. N., Brown, J. K., Ligthart, M. E., Sattar, N., Njualem, D. K., and Kvarnheden, A. 2012. Ageratum conyzoides: A host to a unique begomovirus disease complex in Cameroon. Virus Res. 163:229-237.

13. Li, Z. H., Xie, Y., and Zhou, X. P. 2005. Tobacco curly shoot virus DNA $\beta$ is not necessary for infection but intensifies symptoms in a host-dependent manner. Phytopathology 95:902-908.
14. Mansoor, S., Briddon, R. W., Zafar, Y., and Stanley, J. 2003. Geminivirus disease complexes: An emerging threat. Trends Plant Sci. 8:128-134.

15. Saunders, K., Bedford, I. D., Briddon, R. W., Markham, P. G., Wong, S. M., and Stanley, J. 2000. A unique virus complex causes ageratum yellow vein disease. P. Natl. Acad. Sci. USA. 97:6890-6895.

16. Saunders, K., Briddon, R. W., and Stanley, J. 2008. Replication promiscuity of DNA- $\beta$ satellites associated with monopartite begomoviruses; deletion mutagenesis of the Ageratum yellow vein virus DNA- $\beta$ satellite localizes sequences involved in replication. J. Gen. Virol. 89:3165-3172.

17. Saunders, K., Norman, A., Gucciardo, S., and Stanley, J. 2004. The DNA $\beta$ satellite component associated with ageratum yellow vein disease encodes an essential pathogenicity protein ( $\beta C 1)$. Virology 324:37-47.

18. Shigenori, U., Masatoshi, O., Masataka, Y., and Yoichi, Y. 2012. Pathogenicity and insect transmission of a begomovirus complex between Tomato yellow leaf curl virus and Ageratum yellow vein betasatellite. Virus Genes 44:338-344.

19. Xie, Y., Jiang, T., and Zhou, X. P. 2006. Agroinoculation shows Tobacco leaf curl Yunnan virus is a monopartite begomovirus. Eur. J. Plant Pathol 115:369-375.

20. Xie, Y., Zhou, X. P., Zhang, Z. K., and Qi, Y. J. 2002. Tobacco curly shoot virus isolated in Yunnan is a distinct species of Begomovirus. Chinese Sci. Bull. 47:197-200.

21. Xiong, Q., Guo, X. J., Che, H. Y, and Zhou, X. P. 2007. Ageratum yellow vein China virus is a distinct Begomovirus species associated with DNAbeta molecule. Phytopathology 97:405-411.

22. Zhang, H., Ma, X. Y., Qian, Y. J., and Zhou, X. P. 2010. Molecular characterization and pathogenicity of Papaya leaf curl China virus infecting tomato in China. J. Zhejiang Uni. 11:109-114.

23. Zhou, X. P., Xie, Y., Peng, Y., and Zhang, Z. K. 2003. Malvastrum yellow vein virus, a new Begomovirus species associated with satellite DNA molecule. Chinese Sci. Bull. 48:2205-2209.

24. Zhou, X. P., Xie, Y., and Zhang, Z. K. 2001. Molecular characterization of a distinct begomovirus infecting tobacco in Yunnan, China. Arch. Virol. $146: 1599-1606$ 